\title{
A Stem Cell Harvesting Manipulator with Flexible Drilling Unit for Bone Marrow Transplantation
}

\author{
Kota Ohashi ${ }^{1}$, Nobuhiko Hata ${ }^{1}$, Tomoko Matsumura ${ }^{2}$, \\ Naoki Yahagi ${ }^{2}$, Ichiro Sakuma ${ }^{3}$, and Takeyoshi Dohi ${ }^{1}$ \\ ${ }^{1}$ Graduate School of Information Science and Technology, the University of Tokyo, \\ 7-3-1 Hongo Bunkyo-ku Tokyo 113-8656, Japan \\ \{kotaoha, noby, dohi\}@atre.t.u-tokyo.ac.jp \\ http://www.atre.t.u-tokyo.ac.jp \\ ${ }^{2}$ Graduate School of Medicine, the University of Tokyo, \\ 7-3-1 Hongo Bunkyo-ku, Tokyo 113-8656, Japan \\ Naokiyah@aol.com, tmatsumu-tky@umin.ac.jp \\ ${ }^{3}$ Graduate School of Frontier Sciences, the University of Tokyo, \\ 7-3-1 Hongo Bunkyo-ku, Tokyo 113-8656, Japan \\ isakuma@k.u-tokyo.ac.jp \\ http://miki.pe.u-tokyo.ac.jp/lab.html
}

\begin{abstract}
We present the development of an innovative device (Stem Cell Harvesting Manipulator) to get hematopoietic stem cells for bone marrow transplantation. By using this manipulator, stem cells are harvested from the iliac bone of the donor with minimal puncture. Additionally, the time for carrying out harvesting and the contamination of $\mathrm{T}$ cells can be minimized. In this paper, we report the development of a prototype of the Stem Cell Harvesting Manipulator with Flexible Drilling Unit. The manipulator is inserted into the medullary space from the iliac crest and aspirates the bone marrow while an end-mill on the tip of the Flexible Drilling Unit drills through cancellous bone to create a curved path. We found that the manipulator can be inserted into the medullary space of the pig iliac bone $131 \mathrm{~mm}$ by $32.1 \mathrm{~mm} / \mathrm{min}$ and harvests a phantom of bone marrow about 6 times as much in mass as the conventional Aspiration Method. Further consideration regarding whether or not this device can harvest viable hematopoietic stem cells should be considered through experiments using laboratory animals.
\end{abstract}

\section{Introduction}

Over the past three decades, Bone Marrow Transplantation (BMT) has been established as a curative form of therapy for various acquired hematological malignancies and congenital immune deficiencies such as leukemia or lymphoma [1,2]. On the other hand, the number of cases of BMT has been increasing so rapidly that it has become a problem to harvest hematopoietic stem cells from donors' bone marrow in the iliac bone invasively by means of the conventional "Aspiration Method" using bone marrow needles [3]. Though animal studies have been made on harvesting 
methods from the long bone [4,5], alternatives to the Aspiration Method, how to collect stem cells from a donor's iliac bone, have not been examined to this time.

Briefly stated, there are problems with the"Aspiration Method": the invasiveness of punctures by bone marrow needles and the low density of harvested graft [6]. Doctors generally aspirate about $10 \mathrm{cc}$ of bone marrow to be used as graft with each puncture and totally 500-1200cc from donors' iliac bone. That is to say, donors have to be punctured about 50-120 times by a series of harvest. Moreover, the density of harvested graft is so low that doctors aspirate much more graft than required. In other words, the harvested graft includes much contamination from peripheral blood, which contains greater than $20 \%$ of $\mathrm{T}$ cells that can cause acute graft-versus-host disease (GVHD, a sort of acute rejection) [3].

To tackle the issues, we propose a device (Stem Cell Harvesting Manipulator) to approach medullary space with flexible drill to create a curved path (Flexible Drilling Unit) and harvest graft using negative pressure generated by an actuator. Flexible Drilling Unit like catheter is inserted into medullary space of the iliac bone through a bone marrow needle as a trochanter while an end-mill on the tip of the unit drilled cancellous bone to create a curved path. Then Stem Cell Harvesting Manipulator generates negative pressure to harvest viscous bone marrow efficiently that contains stem cells (Fig.1). By using this method, dense hematopoietic stem cells are harvested within minimal puncture.

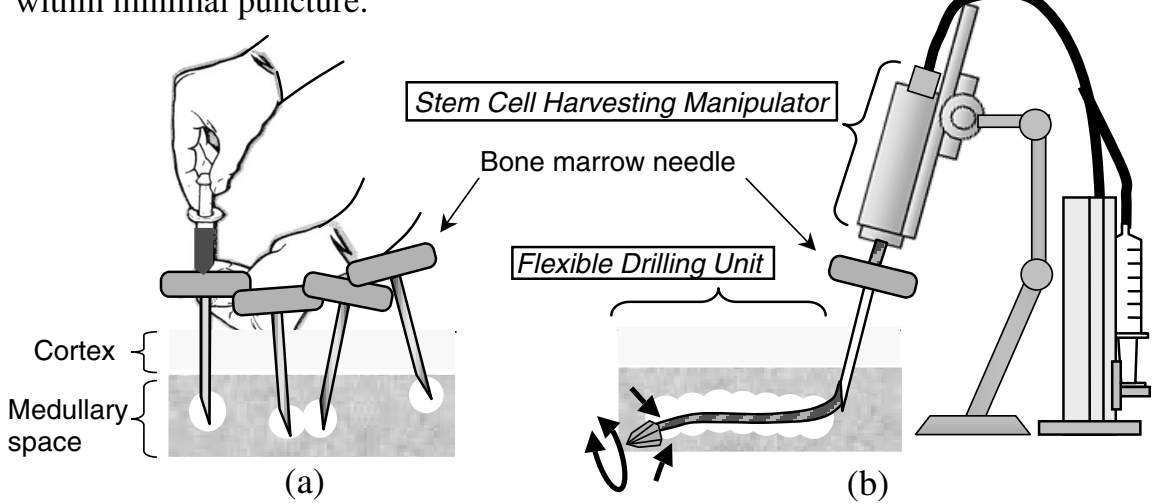

Fig. 1. Concept of the "Stem Cell Harvesting Manipulator" vs. the conventional "Aspiration method" (a) Aspiration Method [7]: To aspirate viscous bone marrow, doctors pull the syringe connected to the bone marrow needle as fast as possible to generate negative pressure. Stem cells only around the tip of the needle, however, can be harvested by a puncture. So, doctors have to puncture for many (50-120) points. (b) A new method for stem cell harvesting using "Stem Cell Harvesting Manipulator": First, the end-mill on the tip of the Flexible Drilling Unit is inserted into medullary space and drills cancellous bone to create curved path. Then, the unit is inserted through the path and harvests graft

The clinical contribution of this study is to minimize invasiveness of donors by means of harvesting denser graft from wider area of the iliac bone with minimal punctures. Additionally, patients and doctors take benefits because risks of the GVHD and time needed for harvesting can be minimized. 
The engineering contribution of this study is to develop a small device to drill into cancellous bone while creating a curved path with the Flexible Drilling Unit, and to generate negative pressure enough to harvest viscous bone marrow efficiently.

First of all, we have produced a prototype of Stem Cell Harvesting Manipulator with Flexible Drilling Unit and carried out in vitro experiments for evaluation using the iliac bone of the pig.

\section{Methods}

\subsection{System Requirements}

To design the prototype of the Stem Cell Harvesting Manipulator, we referred to a model of adult female human iliac bone. It is 5 to $24 \mathrm{~mm}$ in thickness and $153 \mathrm{~mm}$ in width. In order to insert the Flexible Drilling Unit, the unit is limited to less than 5 $\mathrm{mm}$ in diameter and more than 100-150 $\mathrm{mm}$ in length. The radius of curvature of the iliac bone is more than $60 \mathrm{~mm}$.

The iliac bone is so curved that the Flexible Drilling Unit has to create a curved path; therefore the unit has to be flexible. Though a variety of new surgical tools with active bending mechanism are currently under development as part of computer assisted surgery (CAS) and stand-alone devices [8-13], it is not necessary for this unit to bend actively controlled by doctors because of the structure of the iliac bone. Once the unit reaches through the bone marrow needle to medullary space where stem cells are stored, the unit can move within only medullary space enclosed by cortical bone that is hard to drill through. In other words, cortical bone works as a guide.

\subsection{System Overview}

The prototype of Stem Cell Harvesting Manipulator consists of three units, the Stem Cell Harvesting Manipulator itself, the Flexible Drilling Unit and the power unit (Fig.2). The power unit generates negative pressure to harvest stem cells and rotation to drill cancellous bone. The Stem Cell Harvesting Manipulator is attached to an retainer that is generally used for clinical application. Harvested graft from the tip of the Flexible Drilling Unit is collected into a bag with an anticoagulant. Actuators on the power unit and an optical fiber photoelectric switch on the manipulator are controlled by a stored programmable motion controller (EMP2002-V1, Oriental Motor, Japan).

\subsection{Flexible Drilling Unit}

A flexible rod made of high carbon steel $0.8 \mathrm{~mm}$ in diameter transmits rotation to the end-mill on the tip of the Flexible Drilling Unit (Fig.3a). A liner tube made of helical stainless sheet and coated with PVC operates as a path of harvested graft. To drill through fragile cancellous bone, we selected a tip of the self-drilling screw as the endmill $3.5 \mathrm{~mm}$ in diameter (Fig.3b). In order to generate strong flow on the tip of it, inner diameter of the tube is $2.2 \mathrm{~mm}$, much larger than normal bone marrow needles, $1.6 \mathrm{~mm}$. The length is $250 \mathrm{~mm}$, total length of a bone marrow needle and insertion. 


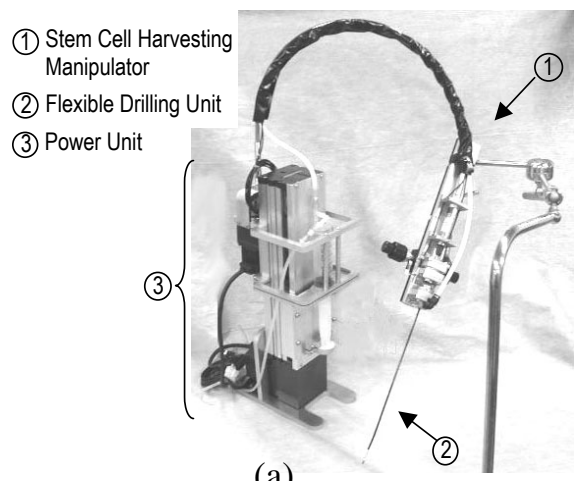

(a)

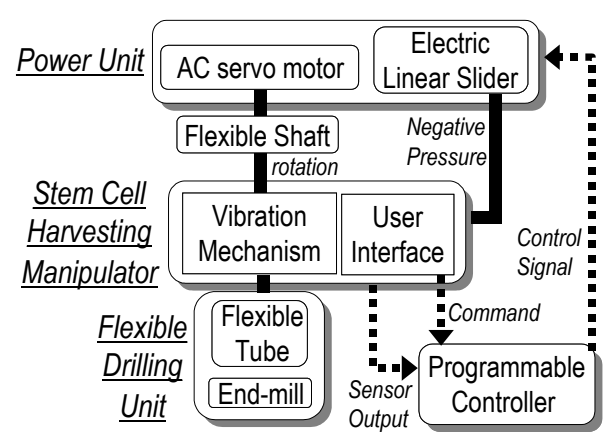

(b)

Fig. 2. The prototype of Stem Cell Harvesting Manipulator: (a) Overview of the system: (b) System configuration: it consists of three units, the Stem Cell Harvesting Manipulator, the Flexible Drilling Unit and a power unit controlled by stored programmable controller

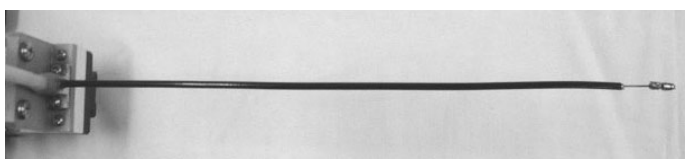

(a)

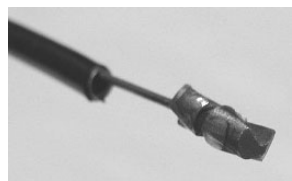

(b)

Fig. 3. The Flexible Drilling Unit: (a) overview: $250 \mathrm{~mm}$ in length and $3.5 \mathrm{~mm}$ in diameter (b) the end-mill on the tip of the unit: It rotates and vibrates axially. Graft is harvested from an aperture between flexible tube and the rod of the end-mill

\subsection{Stem Cell Harvesting Manipulator}

There are mainly two functions in the Stem Cell Harvesting Manipulator (Fig.4a). One is transmission of rotation and negative pressure from the power unit to the Flexible Drilling Unit. The other is user-interface to control linear movement of the manipulator and collection of graft manually by doctors. The rotation from the power unit transmits to the end-mill through the intermediary of the vibration mechanism (Fig.4b).

By means of collisions between hammering parts of the mechanism, the end-mill vibrates axially $16.7 \mathrm{~Hz}$ by $250 \mathrm{rpm}$ to crush and drill cancellous bone. Additionally, not to drill through cortical bone accidentally and injure internal organs, the mechanism doesn't transmit power until the end-mill receive valid axial counter force from cancellous bone detected by an optical fiber photoelectric sensor.

\subsection{The Power Unit for Negative Pressure and Drilling}

To generate negative pressure, the power unit contains an electric linear slider. The slider is equipped with a 60cc syringe normally used in clinical field and pulls it like a syringe pump. The stroke of the slider is $100 \mathrm{~mm}$. A tube to transmit negative pres- 
sure is connected from the syringe to the Stem Cell Harvesting Manipulator through check valves not to generate backward flow accidentally.

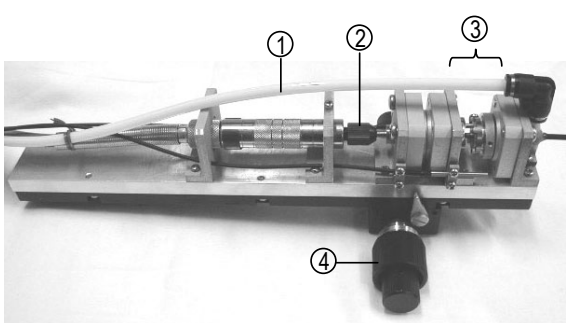

(a)

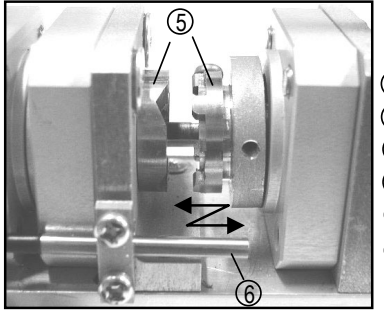

(b)
(1) tube

(2) flexible shaft

(3) vibration mechanism

(4) manual linear stage

(5) hammering parts

(6) optical fiber sensor

Fig. 4. the Stem Cell Harvesting Manipulator: (a) overview: the manipulator is fixed on a linear stage the stroke of which is $300 \mathrm{~mm}$. (b) the vibration mechanism: By means of collisions between hammering parts, the end-mill vibrates axially to crush and drill cancellous bone

The power unit also generates rotation for end-mill by an AC servo-motor. The shaft of the motor is connected with the vibration mechanism of the Manipulator through the intermediary of a flexible shaft that is $800 \mathrm{~mm}$ in length and $8.0 \mathrm{~mm}$ in diameter.

\section{$3 \quad$ Results}

\subsection{In vitro Experiment of Drilling}

We had experiments for evaluation about mechanical performance of drilling using iliac bone of the pig, which is similar to that of human in respect of cutting [14]. The prototype was inserted into medullary space from a spot in the iliac crest.

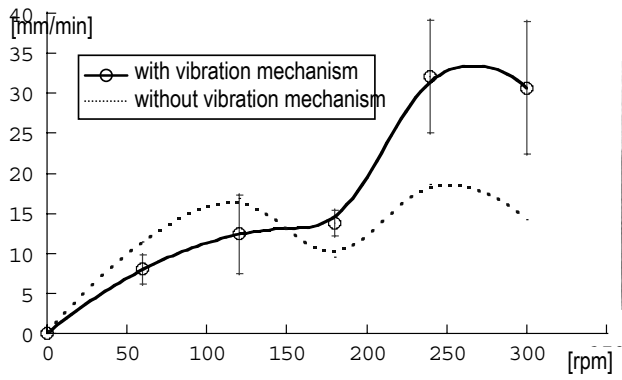

(a)

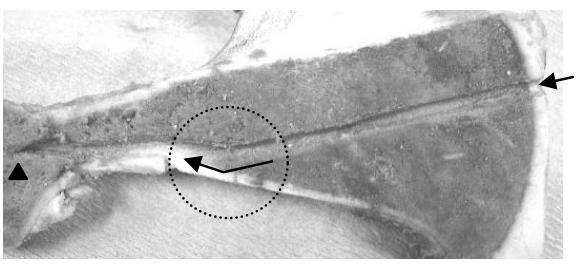

(b)

Fig. 5. Results of experiments for evaluation about mechanical performance of drilling using iliac bone of the pig: (a) the relation between rotation speed and the velocity of insertion: It is found that drilling with the vibration mechanism (solid line) had an advantage of that without the mechanism (dotted line) in velocity of insertion especially over $200 \mathrm{rpm}$. (b) a cross section of the curved path created by the Flexible Drilling Unit: It is $131 \mathrm{~mm}$ in length and $3.9 \mathrm{~mm}$ in diameter. The turning point (dotted circle) can be seen 
Firstly, we measured the relation between the velocity of insertion and rotation speed both with and without the vibration mechanism (Fig.5a). The velocity rose continuously and slightly till $200 \mathrm{rpm}$ and rose rapidly between $200-250 \mathrm{rpm}$. Additionally, it is found that drilling with vibration mechanism had an advantage of that without the mechanism in velocity of insertion especially over $200 \mathrm{rpm}$.

Secondly, we tried inserting the unit into medullary space as deep as possible and measure the depth to estimate whether the unit can drill cancellous bone and approach wide area of iliac bone (Fig.5b). It was found that the unit could create a path over $75.0 \mathrm{~mm}$ to $131 \mathrm{~mm}$ in length while cortical bone worked as a guide and changed the direction of the end-mill. The path connected from the point of the iliac crest to the acetabulum.

\subsection{Phantom Experiment of Harvesting}

We had experiments for evaluation about mechanical performance of harvesting using agar as a phantom of the bone marrow. The phantom was harvested by two methods, the Aspiration Method using bone marrow needle and our method using the Stem Cell Harvesting Manipulator (Fig.6). Then, We measured both of the weight of the harvested phantom. As a result, the graft harvested by a puncture is $3.10 \mathrm{~g}$ by Aspiration Method on average and $18.8 \mathrm{~g}$ by our method.

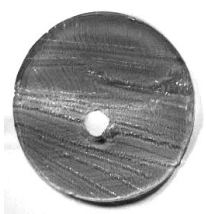

(a)

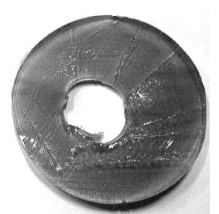

(b)

Fig. 6. Comparison of the harvested phantoms (made of agar) between conventional Aspiration Method and Stem Cell Harvesting Manipulator: (a) the phantom harvested by the Aspiration Method: It is $8.54 \mathrm{~mm}$ in diameter and $3.10 \mathrm{~g}$ in weight. (b) the phantom harvested by the Stem Cell Harvesting Manipulator: It is $20.4 \mathrm{~mm}$ in diameter and $18.8 \mathrm{~g}$ in weight on average. That is, the manipulator can harvest graft from wider area

\section{Discussion}

\subsection{Mechanical Performance}

On the basis of the results of the experiments, it is found that the prototype has possibility to drill into iliac bone. As I mentioned before, the width of human iliac bone is less than about $150 \mathrm{~mm}$, enough for the prototype to create curved path. Additionally, the vibration mechanism on the Flexible Drilling Unit showed adequate performance as a drilling mechanism for iliac cancellous bone though there was much dispersion. To optimize the mechanism for drilling, further study about modeling of interaction between end-mill and bone [15]. 
According to the experiment of harvesting, it can be said that the new method has capacity to harvest graft from wider area; however, we must take much consideration about the difference of mechanical behavior between the phantom made of agar and real bone marrow. In addition, stem cell is stored in vacancies of cancellous bone, much different from the phantom. We will reconsider these points and optimize the mechanical performance through animal experiments.

\subsection{Perspective}

Further consideration should be given whether our new method using the Stem Cell Harvesting Manipulator can collect viable hematopoietic stem cells enough to be used as graft for bone marrow transplantation. To confirm it, we should test the harvested graft for some biological tests by means of colony assay and flow cytometry.

It is true that peripheral blood stem cell transplantation (PBSCT, harvesting stem cells from peripheral blood) has been increasing, but some troubles about donors have been reported recently [3]. On the other hand, methods for stem cell ex vivo expansion are developing [16,17], but they were still used for few cases so far. Indeed, the Stem Cell Harvesting Manipulator would work in cooperation with these methods.

Today, the significance of bone marrow cells has been increasing rapidly from the viewpoint of not only cure for immune deficiencies and hematological malignancies [18] but also tissue engineering [19,20]. It can be said that the method for stem cell harvesting will play a more important part in the near future.

\section{Conclusion}

First, we have developed a new method for stem cell harvesting for bone marrow transplantation using an innovative device, Stem Cell Harvesting Manipulator with Flexible Drilling Unit. By using this manipulator, dense stem cells are harvested from iliac bone of the donor within minimal puncture.

Then, we produced a prototype of the manipulator and did experiments for estimation whether it can create curved path and harvest phantom of bone marrow. Through the experiments using pig iliac bone, the manipulator is inserted into the medullary space from the iliac crest $75.0-131 \mathrm{~mm}$ by $32.1 \mathrm{~mm} / \mathrm{min}$, enough to sweep the iliac bone. Moreover, the prototype aspirated the phantom of bone marrow $18.8 \mathrm{~g}$ by one puncture, about six times as much as the conventional Aspiration Method.

It has been clear that the Stem Cell Harvesting Manipulator, inserted into medullary space curvedly using the Flexible Drilling Unit and harvesting stem cells mechanically, have possibility to become an alternative of the Aspiration Method and minimize the invasiveness to donors and patients.

\section{References}

1. E. D. Thomas: Does bone marrow transplantation confer a normal life span., N. Engl. J. of Med. (1999) 341, pp. 50-51 
2. J. M. Rowe, H. M. Lazarus and A. M. Carella: Handbook of Bone Marrow Transplantation, Martin Dunitz, London (1997) vii

3. S. J. Forman, K. G. Blume and E. D. Thomas: Bone Marrow Transplantation, Blackwell Scientific Publications, Boston (1999) ch.21, pp. 259-269

4. T. Kushida, M. Inaba, K. Ikebukuro, T. Nagahama, H. Oyaizu, H. Lee, T. Ito, N. Ichioka, H. Hisha, K. Sugiura, S. Miyashima, N. Ageyama, F. Ono, H. Iida, R. Ogawa and S. Ikehara: "A New Method for Bone Marrow Cell Harvesting", Stem Cells, Vol. 18, No.6 (2000) pp. 453-456

5. K. Ohashi, T. Shibata, N. Hata, N. Yahagi, T. Matsumura, E. Kobayashi, I. Sakuma and T. Dohi: "Development of Minimally Invasive Bone Marrow Cell Harvester", Proc. of The 10th Meeting of JSCAS (2001) pp. 101

6. H. J. Deeg, H. -G. Klingemann, G. L. Phillips and G. Van Zant: A Guide to Blood and Marrow Transplantation 3rd edn, Springer-Verlag, Berlin Heidelberg (1999) ch. 2, pp. 7580

7. Islam: Manial of Bone Marrow Examination, harwood academic publishers, Amsterdam (1990) ch. 2, pp. 5-29

8. P. Dario: A Novel Mechatronic Tool for Computer-Assisted Arthroscopy, IEEE Trans. on Information Technology in Biomedicine Vol.4, No.1 (2000) pp. 15-28

9. R. Nakamura, T. Oura, E. Kobayashi, I. Sakuma, T. Dohi, N. Yahagi, T. Tsuji, D. Hashimoto, M. Shimada and M. Hashizume: Multi-DOF Forceps Manipulator System for Laparoscopic Surgery, Proc. of 4th MICCAI (2001) pp. 606-613

10. P. Dario, C. Paggetti, N. Troisfontaine, E. Papa, T. Ciucci, M. C. Carrozza and M. Marcacci: A miniature steerable end-effector for application in an integrated system for computer-assisted arthroscopy, Proc. of ICRA Vol.2 (1997) pp. 1573-1579

11. T. Fukuda, S. Guo, K. Kosuge, F. Arai, M. Negoro and K. Nakabayashi: Micro active catheter system with multi degrees of freedom, Proc. of ICRA (1994) pp. 2290-2295

12. H. Rininsland: ARTEMIS. A telemanipulator for cardiac surgery, Europian J. of Cardiothoracic Surgery 16 Suppl.2, (1999) pp. s106-s111

13. K. Ikuta, T. Kato, S. Nagata: Micro active forceps with optical fiber scope for intra-ocular microsurgery, Proc. of the IEEE MEMS (1996) pp. 456-461

14. S. Itoh: Bull. of Japan Society of Mechanical Engineers 26-222 (1983) pp. 2295

15. V. G. Kaburlasos, V. Petridis, P. Brett and D. Baker: Learning a linear association of drilling profiles in stapedotomy surgery, Proc. of ICRA Vol.1 (1998) pp. 705-709

16. J. Jaroscak: A phase 1 trial of augment of unrelated umbilical cord blood transplantation with ex-vivo expanded cells, Blood Suppl.1, (1998) pp. 646a

17. X. Sui: gp130 and c-Kit signalings synergize for ex vivo expansion of human primary hematopoietic progenitor cells, Proc. of Natl. Acad. Sci. USA 92 (1995) pp. 2859-2863

18. M. Marmont: Immune ablation followed by allogeneic or autologous bone marrow transplantation: a new treatment for severe autoimmune diseases? , Stem Cells Vol.12 (1994) pp. 125-135

19. J. Kohyama, H. Abe, T. Shimazaki, A. Koizumi, K. Nakashima, S. Gojo, T. Taga, H. Okano, J. Hata and A. Umezawa: Brain from bone: efficient "meta-differentiation" of marrow stroma-derived mature osteoblasts to neurons with Noggin or a demethylating agent. , Differentiation Oct;68 (4-5) (1996) pp. 235-44

20. P. Bianco, M. Riminucci, S. Gronthos, PG. Robey: Bone marrow stromal stem cells: nature, biology, and potential applications, Stem Cells, 19(3) (2001) pp. 180-92 